\title{
The biological and prognostic significance of angiotropism in uveal melanoma
}

\author{
Raymond L Barnhill ${ }^{1,2}$, Mengliang Ye ${ }^{3}$, Aude Batistella ${ }^{4}$, Marc-Henri Stern ${ }^{4}$, Sergio Roman-Roman ${ }^{3}$, Rémi Dendale ${ }^{5}$, \\ Olivier Lantz ${ }^{6}$, Sophie Piperno-Neumann ${ }^{7}$, Laurence Desjardins ${ }^{8}$, Nathalie Cassoux ${ }^{2,8}$ and Claire Lugassy ${ }^{3}$
}

Angiotropism is a marker of extravascular migration of melanoma cells along vascular and other structures and a prognostic factor in cutaneous melanoma. Because of this biological and prognostic importance in cutaneous melanoma, angiotropism was studied in uveal melanoma (UM). This retrospective study performed at a single ocular oncology referral center included 89 patients from the study period 2006-2008. All patients were diagnosed with UM from the choroid and/or ciliary body. All patients underwent enucleation for prognostic purposes and definitive therapy. Clinical, histopathological, and molecular variables included patient age, gender, extraocular extension, tumor location (ciliary body or not), optic nerve invasion, angiotropism, neurotropism, melanoma cell type, BAP1 mutation, and monosomy 3. Angiotropism was defined as melanoma cells arrayed along the abluminal vascular surfaces without intravasation in the sclera and/or episcleral tissue. The study included 51 women (57.3\%) and 38 men with mean and median age: 63 years (range: 25-92). Mean follow-up was 4.4 years (range: 0.2 to 11). Fifty-three (59.6\%) patients developed metastases and 48 (53.9\%) were dead from metastases at last follow-up. Other principal variables recorded were angiotropism in $43.8 \%$, extraocular extension in 7.9\%, epithelioid/mixed cell type in 73.1\%, BAP1 mutation in 41.3\%, and monosomy 3 in $53.6 \%$ of cases. On multivariate analysis, extraocular extension, angiotropism, and monosomy 3 were predictive of metastasis, whereas tumor diameter, epithelioid cell type, angiotropism, and monosomy 3 were predictive of death. Chi-square test confirmed an association between angiotropism and metastasis and death but none with BAP1 mutation and monosomy 3. In conclusion, angiotropism and monosomy 3 were independent prognostic factors for both metastases and death in UM. However, irrespective of any prognostic value, the true importance of angiotropism is its biological significance as a marker of an alternative metastatic pathway.

Laboratory Investigation (2017) 97, 746-759; doi:10.1038/labinvest.2017.16; published online 27 February 2017

Primary uveal melanoma (UM) originates from neural crestderived melanocytes that migrate to the choroid, ciliary body, and iris of the intraocular uveal tract. Although considerable progress has been made in detecting and treating UM, mortality rates for UM have remained unchanged over the past 50 years. ${ }^{1-3}$ Despite successful therapy of primary lesions, approximately $30 \%$ of patients ultimately develop fatal metastatic disease. In contrast to cutaneous melanoma, which usually initially spreads to the regional lymph node basin, UM metastases rather prototypically first appear in the liver. ${ }^{1-3}$ This latter phenomenon, while at least partially ascribed to the absence of demonstrable lymphatics within the uveal tract, has otherwise never been adequately explained. A comprehensive understanding of the metastatic process in UM is of fundamental importance as these hepatic metastases are in general resistant to current biological and chemotherapeutic regimens. ${ }^{1-3}$

Multiple clinical, histopathological, and genomic prognostic factors for metastasis and death in UM have been described over the years and include patient age, tumor diameter, tumor thickness, extraocular tumor extension, ciliary body involvement, melanoma epithelioid cell type, and mitotic rate. ${ }^{4-11}$ Recent studies have demonstrated the importance of molecular alterations in the prognosis of UM. For example, chromosome 3 loss (monosomy 3), 8q gain, and a particular gene expression profile (Class 2) are associated

\footnotetext{
Department of Pathology, Institut Curie, Paris, France: ${ }^{2}$ Faculty of Medicine, University of Paris Réné Descartes, Paris, France: ${ }^{3}$ Department of Translational Research, Institut Curie, Paris, France; ${ }^{4}$ 8830 Inserm Unit, Center for Research, Institut Curie, Paris, France; ${ }^{5}$ Department of Radiation Oncology, Institut Curie, Paris, France; ${ }^{6}$ U932 Inserm Unit, Center for Research, Institut Curie, Paris, France; ${ }^{7}$ Department of Medical Oncology, Institut Curie, Paris, France and ${ }^{8}$ Department of Ophthalmology, Institut Curie, Paris, France

Correspondence: Professor RL Barnhill, MD, MSc, Department of Pathology, Institut Curie, 26 rue d'Ulm, Paris cedex 05 75248, France.

E-mail: raymond.barnhill@curie.fr
}

Received 27 December 2016; revised 17 January 2017; accepted 20 January 2017 
Table 1 Clinical and histopathological characteristics in $\mathbf{8 9}$ patients undergoing enucleation for primary uveal melanoma

$\begin{array}{lc}\text { Age (years) } & \text { Median: 63 } \\ & \text { Mean: 62.9 } \\ & \text { Range: 25-92 } \\ \text { Gender } & \text { Women: } 51(57.3 \%) \\ \text { Tumor diameter }(\mathrm{mm}) & \text { Men: } 38 \text { (42.7\%) } \\ & \text { Median: } 19 \\ \text { Tumor thickness }(\mathrm{mm}) & \text { Mean: } 18.5 \\ & \text { Range: 10-26 } \\ & \text { Median: 11.65 } \\ & \text { Mean: 11.5 } \\ \text { Range: 3.9-16.7 }\end{array}$

Metastasis, no. (\%)

Present

$53(59.6 \%)$

Absent

Melanoma-specific mortality, no. (\%)

Dead

Alive

Follow-up, months

Mean

Median

Range

TMN staging

T2: $2 / 89(2.2 \%)$

T3: $22 / 89$ 24.7\%)

T4: $65 / 89$ (73.0\%)

Extraocular extension

Present: 7 (7.9\%)

Absent: 82 (92.1\%)

Angiotropism

Optic nerve invasion

Ciliary body involvement ( $m d=1$ )

Eye laterality

Melanoma cell type Present: 39 (43.8\%) Absent: 50 (56.2\%) Present: 6 (6.7\%)

Absent: 83 (93.3\%)

Present: 11 (12.5\%) Absent: 77 (87.5\%)

Right: 40 (44.9\%)

Left: 49 (55.1\%)

Epithelioid: 24 (27\%) with poor prognosis. ${ }^{3-7}$ More recently, the tumor-suppressor gene BRCA1-associated protein 1 (BAP1) has been mapped to chromosome 3p21.1. ${ }^{6}$ The somatic mutation of BAP1 has been associated with metastatic UM. ${ }^{6,7}$

Relevant to potential metastatic pathways in UM, a newly described interaction between cancer cells and vessels, or more specifically the association of tumor cells with the abluminal surfaces of vascular channels, termed angiotropism, has recently been proposed. ${ }^{12-14}$ Angiotropic tumor cells can spread along the abluminal surfaces of vessels without entering the vascular channels (intravasation), taking the place of pericytes, or pericyte mimicry and therefore making use of the blood vascular network as a track. ${ }^{12}$ This newly described mechanism of tumor cell migration has been termed extravascular migratory metastasis (EVMM) and is believed to represent an alternative means of tumor spread to intravascular dissemination of tumor cells. ${ }^{12-15}$

With respect to UM, it is well known that melanoma cells may traverse trans-scleral emissary channels that contain blood vessels and nerves to reach extraocular structures, and such 'extraocular extension' is an established adverse prognostic factor for patient survival. ${ }^{8-11}$ Coupland et al ${ }^{11}$ observed that apparently all routes of extraocular spread, including aqueous channels, vortex veins, ciliary arteries, and ciliary nerves, analyzed in aggregate correlated with increased mortality. However, the cursory reference to invasion of scleral emissary canals by UM, as an important prognostic factor for the development of metastases by Seddon et al, ${ }^{16}$ may have been among the first observations of the specific phenomenon that we now term 'angiotropism and EVMM'. No specific details were provided in that study. Some authors have maintained that only spread along vortex veins is predictive of increased mortality from metastatic disease. ${ }^{17} \mathrm{It}$ is of interest that a recent report has suggested that intravascular tumor invasion per se is not an important prognostic factor in UM. ${ }^{18}$

Significantly, angiotropism is now recognized as an

Spindle: 24 (27\%)

Mixed: 41 (46.1\%) independent prognostic factor for metastasis and death in cutaneous melanoma. ${ }^{13,14,19-22}$ In particular, angiotropism is predictive of loco-regional and distant melanoma metastases, ${ }^{19}$ a prognostic factor for local and in transit melanoma metastases, ${ }^{20}$ and for microscopic satellite formation and thus is likely to explain the development of such microscopic satellites. ${ }^{22}$ In addition, angiotropism and EVMM have been recently implicated in satellite in transit metastases in rapidly fatal conjunctival melanoma. ${ }^{23}$

Because of the latter considerations, we thought it highly pertinent to study angiotropism in UM. To our knowledge, angiotropism, as defined here and irrespective of the type of

Abbreviation: $\mathrm{md}$, missing data. vascular channels involved, has never been evaluated in primary UM. Herein we report for the first time the significant association of angiotropism with metastasis and death in a series of 89 consecutive enucleations of primary UM. 


\section{MATERIALS AND METHODS}

One hundred and seven consecutive enucleations of primary UM were retrieved from the anatomic pathology database and archives of the Institut Curie from the period 2006-2008. Among this study set, 18 cases were excluded from the study: (1) for 9 cases: glass microslides were not available and (2) for 8 cases, the slides available for review were judged inadequate for assessment, and (3) 1 case upon review was not UM. The final study set constituted 89 cases. This particular cohort of patients (prospectively recorded in a database) contains extensive clinical, histopathological, molecular genetic, and long-term follow-up information. The clinical, histopathological, and outcome data were recorded (Table 1) for each patient. Clinical parameters recorded were as follows: age, gender, eye laterality, anatomical site of the primary melanoma, clinical outcome, and length of follow-up in years.

Formalin-fixed, paraffin-embedded 5-micron sections were prepared for conventional microscopic examination. Histopathological parameters were recorded based on the examination of step sections with a mean 14.5 levels per enucleation (College of American Pathologists Protocol for the Examination of Specimens from Patients with Uveal Melanoma specimenwww.cap.org/apps/docs/committees/ca ncer/cancer_protocols/2012/UvealMelanom_12protocol.pdf). These parameters included greatest tumor thickness (in $\mathrm{mm}$ ), maximum tumor diameter (in $\mathrm{mm}$ ), principal melanoma cell type: epithelioid ( $>90 \%$ epithelioid cells), spindle $(>90 \%$ spindle cells), or mixed ( $>10 \%$ epithelioid cells and $<90 \%$ spindle cells); extraocular extension, optic nerve invasion, location of melanoma: involvement of the ciliary body $v \mathrm{~s}$ other locations, neurotropism, and angiotropism. ${ }^{24}$

Angiotropism was defined as previously described: ${ }^{25,26}$ (1) clearly recognizable (unequivocal) melanoma cells disposed circumferentially, radially, or longitudinally along the abluminal (external) surfaces of the endothelium of microvascular channels (ciliary and Schlemm's vascular channels, and vortex veins in the sclera) and/or episcleral tissue, either in singlelayered or multi-layered arrangements, (2) the latter occurring in at least one or more foci, and (3) no evidence of intravascular melanoma cells. Angiotropism was recorded as present only if it was observed in the sclera distinctly separated from the base of the tumor or in nearby episcleral tissue.

Molecular genetic findings included mutational status for GNAQ, GNA11, BAP1, and monosomy 3 (Table 1), as previously described by Cassoux et al. ${ }^{3}$ GNAQ and GNA11 results are not shown. Because of missing data, the status of chromosomal 8q gain has not been included.

This study was approved by our institutional ethics committee. Written informed consent for the use of tissues and data for research was signed by each patient. The study complied with the principles of the Declaration of Helsinki.

\section{Statistics}

Statistical analysis was performed using the R software V3.1.0 (www.r-project.org). Metastasis-free (disease-free) survival and overall survival rates were estimated by the KaplanMeier method and compared using log-rank test. Differences were considered to be significant when the log-rank $P$-value was $<0.1$. Univariate Cox regression analysis was performed using $P=0.1$ level of significance for the selection of candidate variables. Furthermore, a multivariate Cox regression analysis was performed on all variables remaining significant on univariate regression analysis. A chi-square test was used to examine the association between angiotropism and other prognostic variables.

\section{RESULTS}

\section{Patient and Tumor Characteristics}

The patient clinical information and tumor characteristics are summarized in Table 1. Fifty-one women and 38 men ranging in age from 25 to 92 years ( mean $=62.9$ years; median $=63$ years) comprised the study population.

Primary melanoma histopathological features included laterality: 40 right eyes: 49 left eyes; tumor diameter ranging from 10 to $26 \mathrm{~mm} \quad($ mean $=18.5 \mathrm{~mm}$; $\operatorname{median}=19 \mathrm{~mm})$, tumor thickness range $3.9-16.7 \mathrm{~mm} \quad($ mean $=11.5 \mathrm{~mm}$; median $=11.6 \mathrm{~mm}$ ); TMN classification $(7 \mathrm{th}$ edn.): T2: 2, T3: 22, T4: 65; extraocular extension present in 7 patients; optic nerve invasion in 6 patients; ciliary body involvement in 11/88; and melanoma cell type: epithelioid: 24 , spindle: 24 , mixed: 41 .

Forty-one of the 89 (46.1\%) patients are alive and diseasefree with a follow-up ranging from 1 to 11 years ( mean $=77$ months, median $=84$ months). Fifty-three of the $89(59.6 \%)$ patients developed metastasis during a period ranging from 2 months to 10 years (mean $=46.4$ months, median $=48$ months). Forty-eight $(53.9 \%)$ patients died during a follow-up ranging from 2.4 months to 6 years ( mean $=35.8$ months, median $=36$ months). Finally, at last follow-up 5 patients are alive with metastatic disease.

\section{Angiotropism}

Angiotropism of melanoma cells was recorded in 39 of the 89 $(43.8 \%)$ cases examined. In general, this phenomenon was relatively focal and often observed in only one to two tissue sections. A mean of 14.5 step sections were examined for each enucleation specimen. Angiotropism most commonly involved the ciliary or Schlemm's vascular channels (29 cases $(74.3 \%)$ ) (Figures 1a-d) and less commonly the vortex venous channels (10 cases $(25 \%)$ ), in one or more foci within the sclera and rarely extending to the scleral external surface (extrascleral extension). Usually melanoma cells were disposed as clusters of cells or as multiple layers of cells about the abluminal vascular surfaces. Episcleral micronodular aggregates of melanoma cells were rarely detected in cases with angiotropism and were similar to microscopic or macroscopic satellites/in transit metastases observed in cutaneous and conjunctival melanomas. 

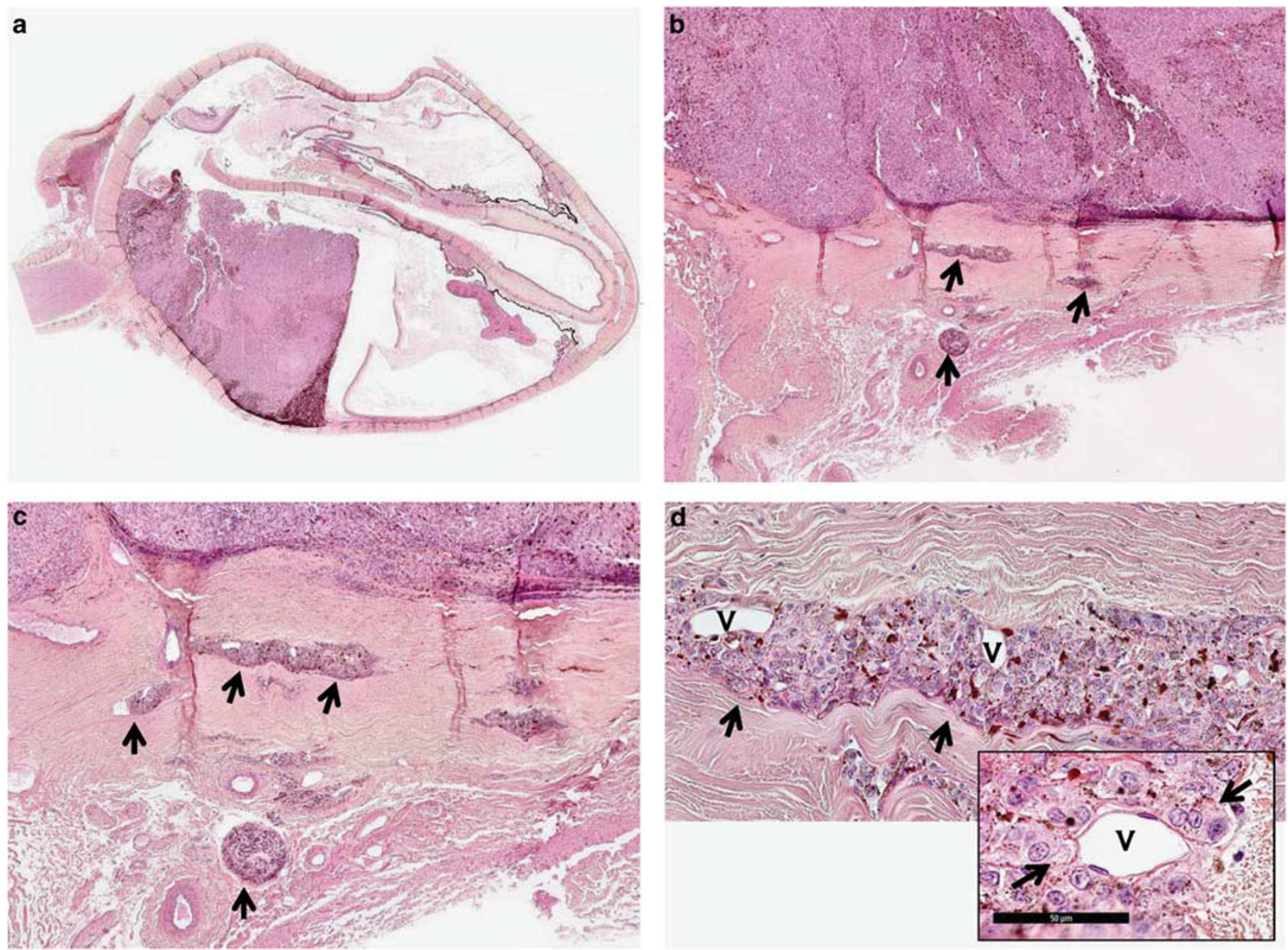

Figure 1 Angiotropism in uveal melanoma. (a) Note large nodular pigmented tumor involving the posterior uveal region. (b) Angiotropism of melanoma (arrow) along posterior ciliary vessels within the sclera at scanning magnification. (c) Melanoma cells are disposed along the abluminal surfaces of the vascular channels (arrows) without intravasation. (d) High magnification shows multi-layered arrangements of pigmented epithelioid melanoma cells (arrows) surrounding this ciliary vascular channel. $V=$ intravascular lumina. Insert: abluminal melanoma cells (arrows) by definition are circumferentially and directly positioned along and in contact with the external surface of this endothelial-lined vascular channel (V). These melanoma cells are in contact with the basement membrane of the endothelial cells. Multi-layering of tumor cells about vascular channels as seen here represents a 'collective' form of cellular migration of tumor cells. ${ }^{27}$

\section{Statistical Analysis}

Based on univariate Cox regression analysis, angiotropism was shown to be one of the most significant prognostic factors for the development of metastases (hazard ratio $(\mathrm{HR})=3.654$, $P<0.0001)$ and death $(\mathrm{HR}=4.89, P<0.0001)$ (Tables 2 and 3), respectively, and $\mathrm{HR}=2.65, P<0.001$ and $\mathrm{HR}=3.41$, $P<0.001$ after adjustment for the other covariates (Tables 4 and 5). HR measures the frequency of an event such as death from UM in a cohort of patients associated with the presence of a particular variable, such as angiotropism $v s$ its absence in a control population of patients with UM during the same period of time.

Variables predictive of metastasis on univariate analysis were extraocular extension, angiotropism, epithelioid cell type, BAP1 mutation, and monosomy 3. Prognostic factors predictive of death were age, tumor diameter, extraocular extension, angiotropism, epithelioid cell type, BAP1 mutation, and monosomy 3 (Figures 2 and 3 ).

In a multivariate analysis for metastasis including only factors significant with univariate analysis $(P<0.1)$, only extraocular extension, angiotropism, and monosomy 3 remained independent (Table 4). Factors predictive of death on multivariate analysis after adjustment for other factors were tumor diameter, angiotropism, epithelioid cell type, and monosomy 3 (Table 5).

A chi-square test confirmed a striking association of angiotropism and metastasis and death (Table 6). Angiotropism was also strongly associated with extraocular extension, epithelioid cell type, neurotropism, and optic nerve invasion. Angiotropism was recorded in 100\% of melanomas manifesting extraocular extension and optic nerve invasion and in $81 \%$ of melanomas with neurotropism. However, 
Table 2 Univariable Cox regression analysis for factors predictive of metastasis

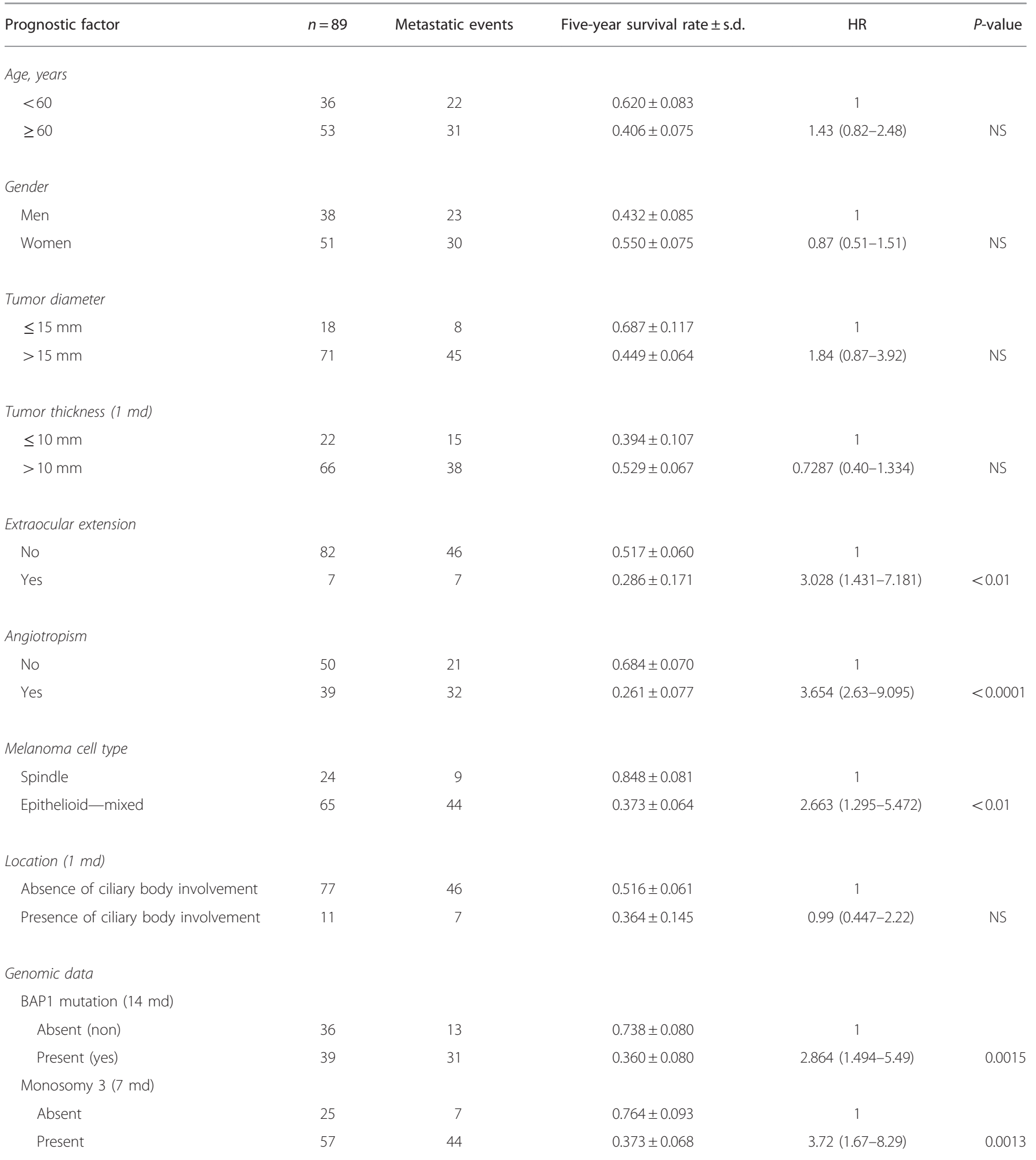

Abbreviations: $\mathrm{HR}$, hazard ratio; $\mathrm{md}$, missing data.

Metastasis rate was estimated by the Kaplan-Meier method and the HR was determined by the Cox model. 
Table 3 Univariable Cox regression analysis for factors predictive of death

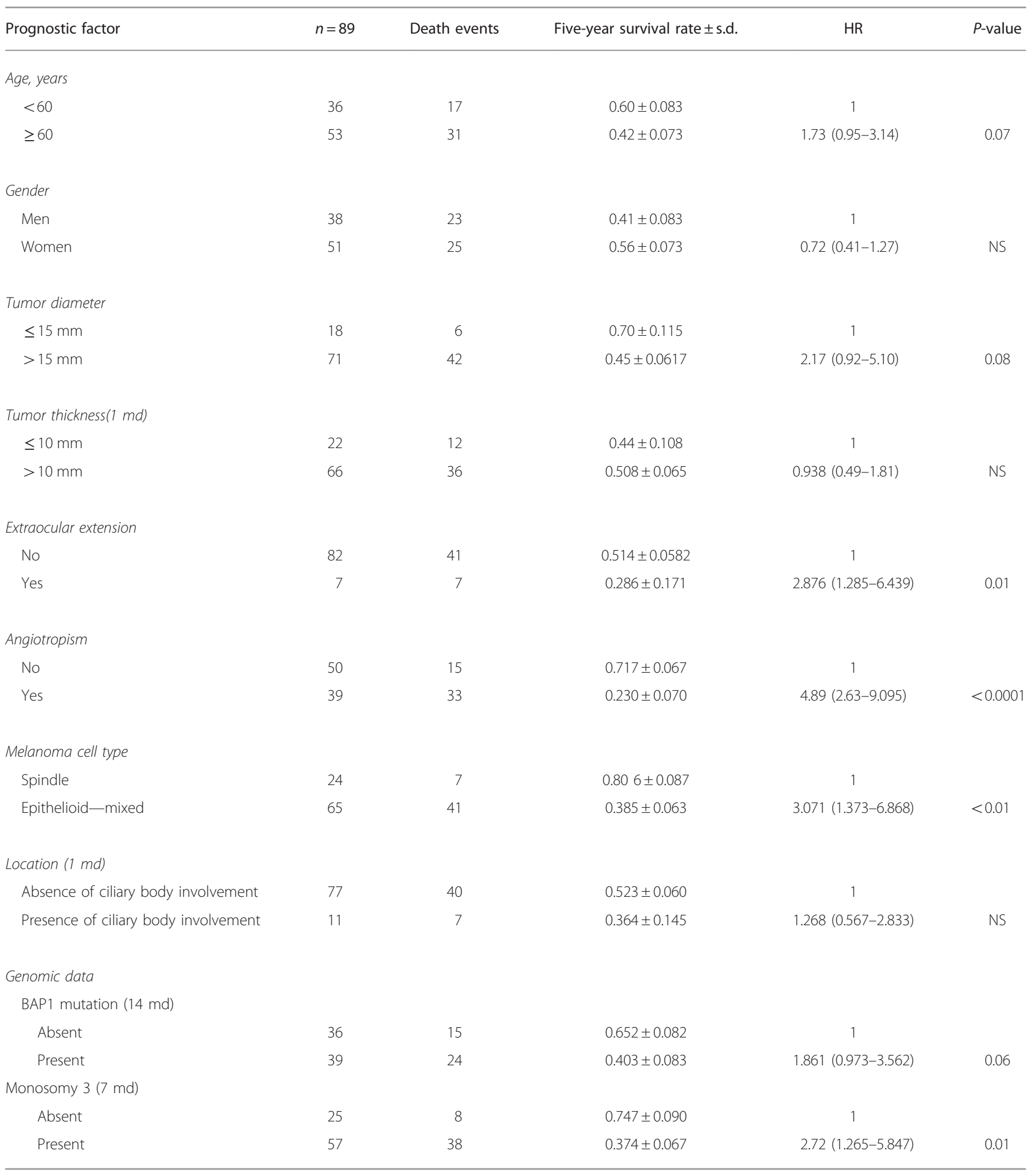

Abbreviations: HR, hazard ratio; md, missing data.

Survival rate was estimated by the Kaplan-Meier method and the HR was determined by the Cox model. 
Table 4 Multivariate Cox regression analysis for factors predictive of metastasis (based on adjustment after univariate regression)

\begin{tabular}{|c|c|c|c|c|}
\hline Prognostic factor & $n=89$ & Metastasis events & $\mathrm{HR}$ & $P$-value \\
\hline \multicolumn{5}{|l|}{ Extraocular extension } \\
\hline No & 82 & 46 & 1 & \\
\hline Yes & 7 & 7 & 3.77 & 0.03 \\
\hline \multicolumn{5}{|l|}{ Angiotropism } \\
\hline No & 50 & 21 & 1 & \\
\hline Yes & 39 & 32 & 2.72 & $<0.001$ \\
\hline \multicolumn{5}{|l|}{ Melanoma cell type } \\
\hline Spindle & 24 & 9 & 1 & \\
\hline Epithelioid_mixed & 65 & 44 & 1.89 & NS \\
\hline \multicolumn{5}{|l|}{ BAP1 mutation (14 md) } \\
\hline Absent (non) & 36 & 13 & 1 & \\
\hline Present (yes) & 39 & 31 & 1.18 & NS \\
\hline \multicolumn{5}{|l|}{ Monosomy 3 (7 md) } \\
\hline Absent & 25 & 7 & 1 & \\
\hline Present & 57 & 44 & 4.53 & 0.01 \\
\hline
\end{tabular}

Abbreviations: $\mathrm{HR}$, hazard ratio; $\mathrm{md}$, missing data.

HR was determined by the Cox model.

angiotropism showed no association with either BAP1 mutation or monosomy 3.

\section{DISCUSSION}

Because of the biological and prognostic significance of angiotropism in cutaneous melanoma, ${ }^{12-15}$ we conducted a comparable study of angiotropism in UM. Our results confirm that angiotropism is strongly associated with the development of metastases and death in UM. Thus, in addition to genomic tumoral characteristics and other established prognostic indicators, ${ }^{3,6,7}$ angiotropism may provide additional prognostic information about risk for metastasis and death in UM patients. However, irrespective of any prognostic value, the true importance of angiotropism is its biological significance as a marker of an alternative metastatic pathway. ${ }^{13-15}$

Although angiotropism as a distinctive microscopic finding has not been systematically studied in UM as to its biological and prognostic significance, observations of angiotropism in the course of analyzing extrascleral or extraocular extension of UM date back many years. In 1962, Starr and Zimmerman ${ }^{8}$ observed that the incidence of extraocular extension of UM ranged from 8 to $40 \%$. In their study of 1842 melanomas of
Table 5 Multivariate Cox regression analysis for factors predictive of death (based on adjustment after univariate regression)

Prognostic factor $\quad n=89 \quad$ Death events HR P-value

$$
\begin{gathered}
\text { Age, years } \\
<60 \\
\geq 60
\end{gathered}
$$

\section{Tumor diameter \\ $\leq 15 \mathrm{~mm}$ \\ $>15 \mathrm{~mm}$}

Extraocular extension

No

Yes

Angiotropism
No
Yes

\section{Epithelioid cell type \\ Spindle}

Epithelioid-mixed

BAP1 mutation (14 md)

Absent

Present

\section{Monosomy 3 (7 md) \\ Absent}

Present
36

53

18

71

82

7

50

39

24

65

65

65

$\begin{array}{rl}7 & 1 \\ 41 & 2.83\end{array}$

2.83

0.04

Abbreviations: $\mathrm{HR}$, hazard ratio; $\mathrm{md}$, missing data. HR was determined by the Cox model.

the choroid and ciliary body, extrascleral extension was recorded in $13 \%$ of cases. They also reported a significantly increased 5-year mortality of $66 \%$ if extraocular extension of tumor was present $v s$ a $33 \%$ death rate if absent. In the course of this retrospective study, the extension of tumor along intrascleral vascular channels (illustrated in a photomicrograph) were discerned in six enucleated melanomas without any evidence of extrascleral extension. These patients subsequently developed orbital recurrences. For us, the latter observations constitute one of the earliest published accounts of angiotropism and its significance in UM.

A more recent comprehensive study of 847 patients addressed all intrascleral routes of extraocular extension as a prognostic factor in UM. ${ }^{11}$ Not only was spread along 
Angiotropism in uvea melanoma

RL Barnhill et al
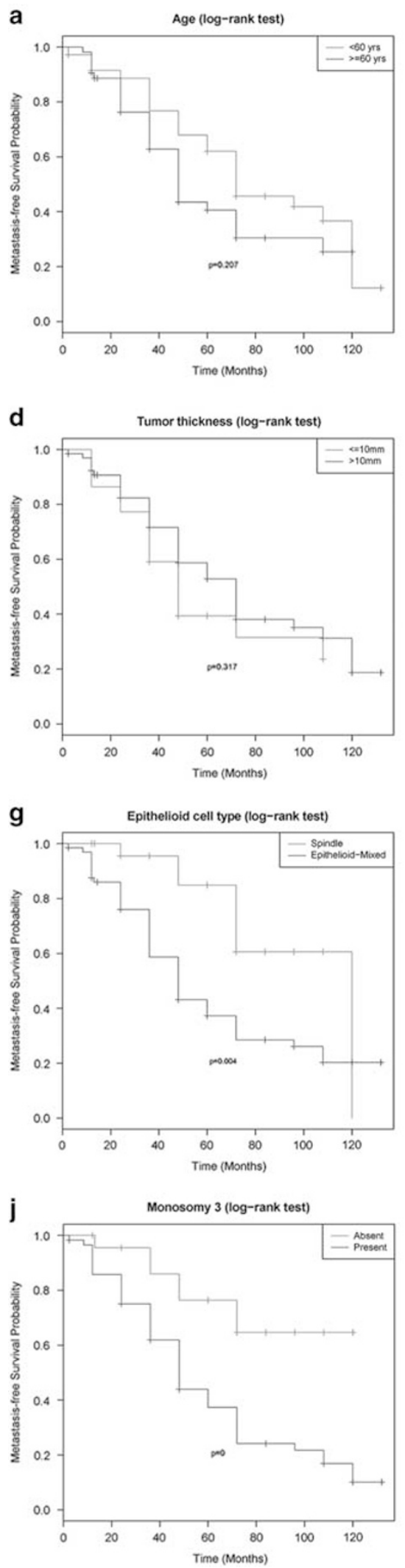
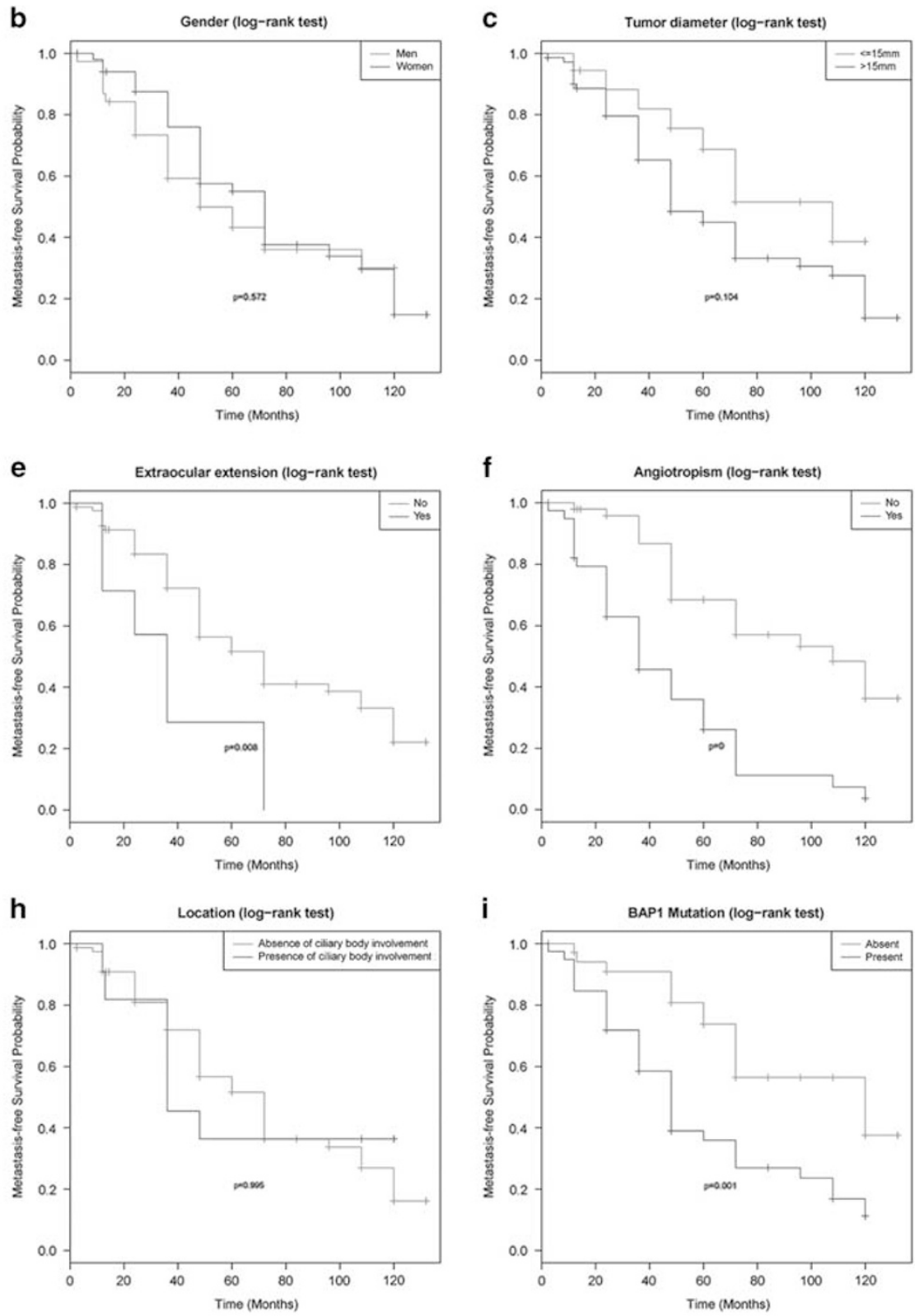

Figure 2 Metastasis-free survival analyzed by the log-rank test for 10 prognostic variables (adj).

www.laboratoryinvestigation.org | Laboratory Investigation | Volume 97 June 2017

753 
a
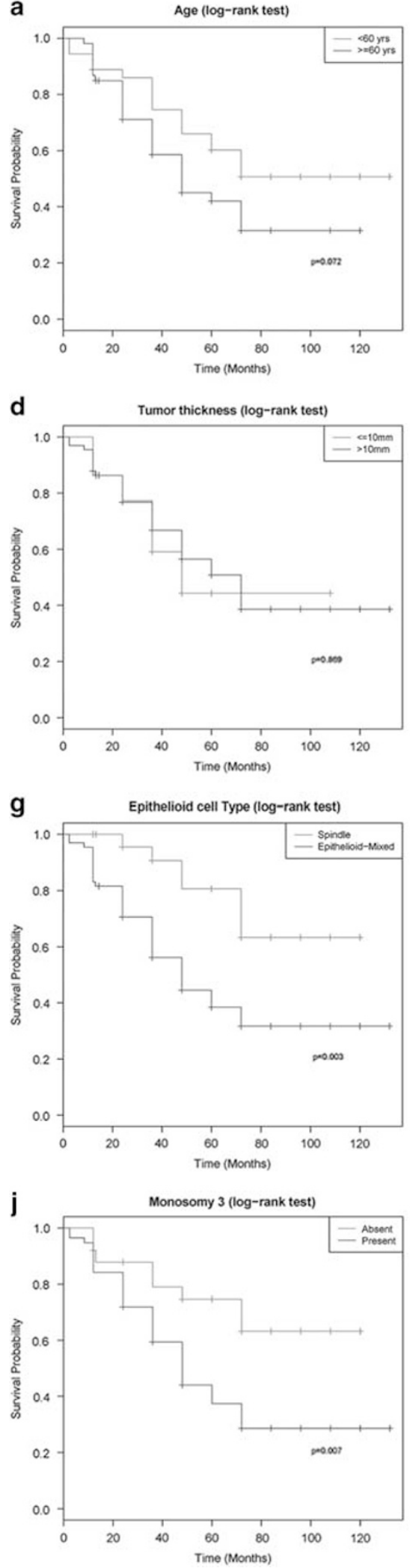

b

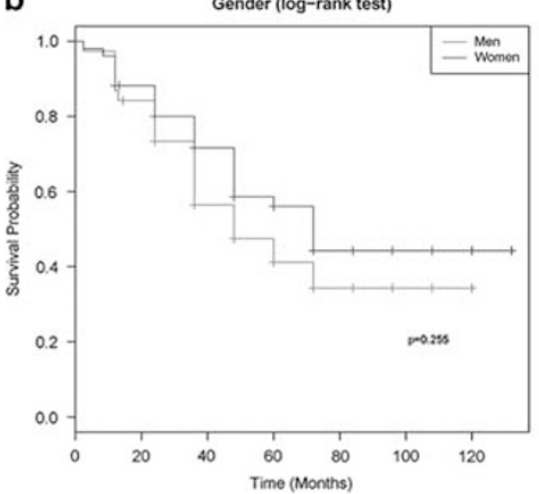

e

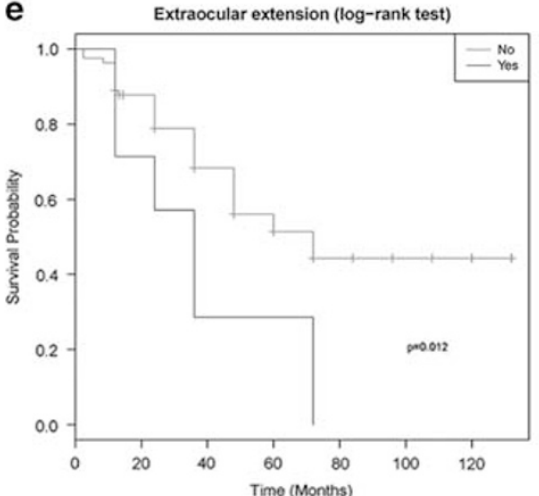

h

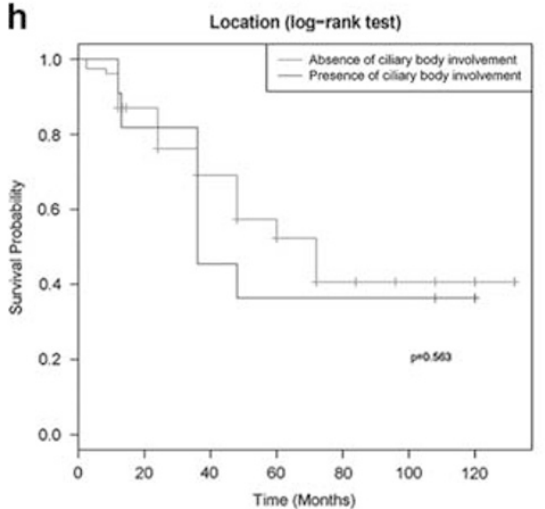

C

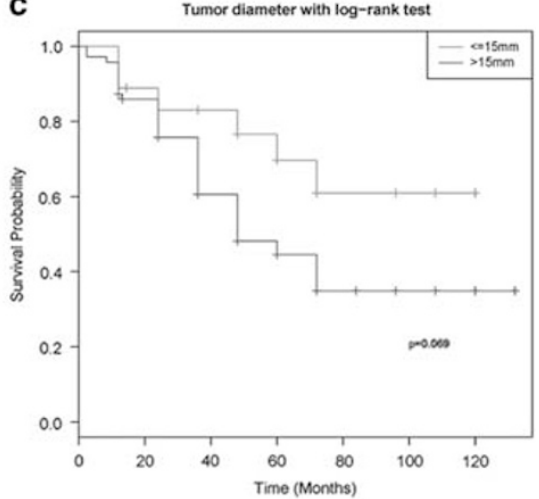

f

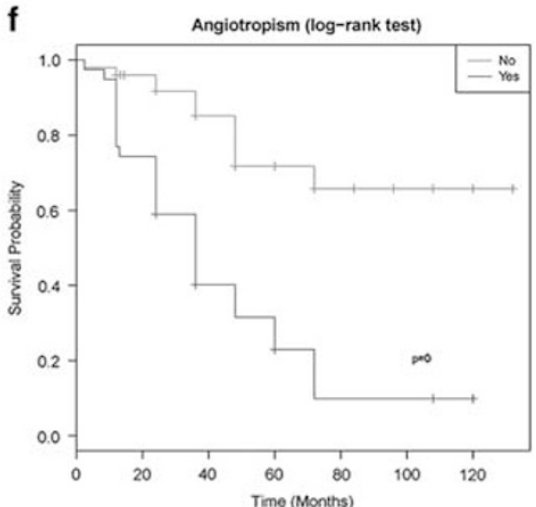

i

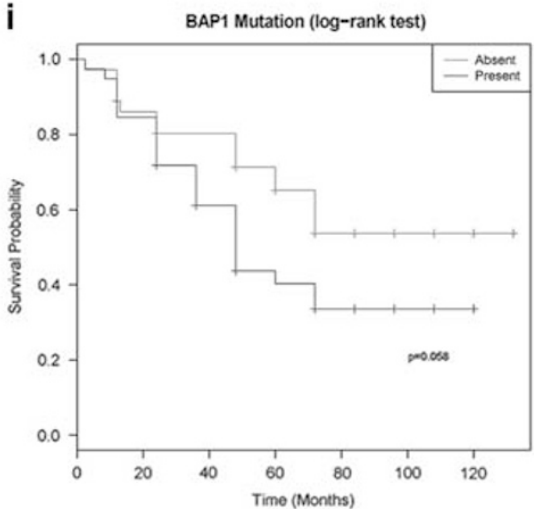

Figure 3 Overall survival analyzed by the log-rank test for 10 prognostic variables $(\mathbf{a}-\mathbf{j})$. 
Table 6 Chi-square test examining association of angiotropism with other prognostic factors

\begin{tabular}{lcc}
\hline Variable & $\begin{array}{c}\text { Angiotropism } \\
\text { present } \\
(n=39)\end{array}$ & $\begin{array}{c}\text { Angiotropism } \\
\text { absent }(n=50)\end{array}$ \\
& & \\
\hline
\end{tabular}

$$
\begin{aligned}
& \text { Death } \\
& \text { Alive }(n=41) \\
& \text { Dead }(n=48)
\end{aligned}
$$

Metastasis

Absent $(n=36)$

Present $(n=53)$

7

32

29

21

Tumor diameter

$\leq 15 \mathrm{~mm}(n=18)$

$>15 \mathrm{~mm}(n=71)$

6

33

12

38

NS

Extraocular extension

Absent ( $n=82)$

Present $(n=7)$

Melanoma cell type

Spindle (24)

Epithelioid-mixed (65)

\section{Neurotropism}

Absent $(n=73)$

Present $(n=16)$

Optic nerve invasion ( $m d=1$ )

Absent $(n=83)$
Present $(n=5)$

\section{BAP1 ( $m d=14$ )}

Absent $(n=36)$
Present $(n=39)$

Monosomy $3(\mathrm{md}=7$ )

$$
\begin{aligned}
& \text { Absent }(n=25) \\
& \text { Present }(n=57)
\end{aligned}
$$

30
NS

27
Abbreviation: $\mathrm{md}$, missing data.

intrascleral vascular channels recorded but also extension along aqueous drainage channels and intrascleral nerves. Although extraocular extension by all routes was found to correlate with metastatic death, the particular type of scleral pathway was judged to be unimportant. These authors concluded that aggressive tumor phenotype and advanced disease were the primary determinates of adverse prognosis rather than extraocular tumor extension per se. In contrast, in a study of $110 \mathrm{UM}$ manifesting extraocular extension as compared with 2148 cases without extraocular disease, Zografos $^{17}$ reported that only extraocular tumor nodules and intrascleral extension of tumor along vortex veins correlated with increased mortality from metastases $v s$ spread via ciliary vascular channels (see below).

The results of the present study suggest that the migration of melanoma cells along vascular and other anatomic structures (EVMM) is potentially an important mechanism of metastasis in UM, as in cutaneous melanoma and other related tumors of neural crest origin such as glioblastoma. ${ }^{12}$ The fact that both cutaneous melanomas and UMs are of neurocristic origin and that neural crest stem cells show comparable extravascular cellular migration in the embryo provide a strong scientific basis for this migratory phenomenon in melanoma. Our findings suggest that all types of vascular channels, ie, the ciliary vessels, vascular channels of Schlemm, and vortex veins, are involved in this process.

One perspective to be considered is that angiotropism in this study simply represents tumor cell migration through trans-scleral (emissary) canals, irrespective of whether vascular structures are present or not. The authors, however, believe that melanoma cell migration along vascular channels (traversing the sclera) is the principal mechanism explaining these observations as the authors have observed similar findings in cutaneous melanoma and in other tumors of neural crest origin such as glioma and glioblastoma. As mentioned above, neural crest stem cells migrate along the abluminal surfaces of vascular channels during at least part of their journey from the neural crest to distant sites in the embryo. In addition, we and others have observed UM spread associated with the canals of Schlemm, which constitute anterior intrascleral (emissary) canals. ${ }^{11}$ Recent observations have confirmed that the canals of Schlemm are in fact unique endothelial-lined channels with combined blood vascular and lymphatic phenotypes and an abluminal basement membrane. ${ }^{27}$ Thus, importantly, it appears that all transscleral canals contain vascular channels, or nerves, or both, and all manifest basement membranes and provide potential routes for angiotropic melanoma cell migration.

One can make the argument that the dense collagen of the reticular dermis of skin is analogous to the sclera as a physical barrier. In both instances, the perivascular spaces (or canals or channels) in both the dermis and the sclera clearly provide routes of lower resistance that could facilitate tumor cell migration. This tumor spread, however, appears to be more specific than simply a migration along an open canal as this migration occurs along the abluminal basement membranes of endothelial cells. The transformation of this normally structured membrane to one that is distinctly amorphous and rich in laminin upon contact with tumor cells strongly suggests fairly specific alterations linked to this migratory 
phenomenon. ${ }^{12}$ As Friedl and Alexander ${ }^{28}$ have emphasized, a vascular basement membrane (and particularly one with laminin alterations) would appear to provide a preferred anatomic structure for tumor cell migration.

In all, $43.8 \%$ of our cases manifested angiotropism; this figure appears roughly comparable to the range of vascular involvement recorded in a previous studies. For example, emissary canal invasion was recorded in $55 \%$ of enucleations in the Collaborative Ocular Melanoma Study, ${ }^{24}$ whereas Coupland et all observed tumor extension along canals of Schlemm in $29.8 \%$, ciliary arteries in $27.4 \%$, and along vortex veins in $18.5 \%$ of enucleations. A number of factors may account for these differences. The results from our study indicate that angiotropism of melanoma cells involving intrascleral vascular channels may be a relatively focal phenomenon and thus sampling error and the number of serial or step sections examined may explain the failure to detect this finding (Wolter ${ }^{29}$ and Barnhill RL, personal observations, 2014-2016). In addition, the increased frequency of angiotropism in our study may be related to the increased lesional diameters of UMs as compared with other studies. ${ }^{11,30}$ The mean basal diameter among our melanomas was 18.5 vs $14.9 \mathrm{~mm}$ in the study by Coupland et al. ${ }^{11}$ As the rate of extraocular extension increases as a function of UM basal diameter and as intrascleral angiotropism and extraocular extension are closely related, the rate of angiotropism should correlate directly with UM lesional diameter. However, such extension of tumor along intrascleral vessels and nerves may be seen in UM of any size. ${ }^{31-34}$ Based on our study with a mean of 14.5 step sections per eye and that of COMS with 15 step sections per case, we recommend sampling of approximately15 step sections per enucleated specimen if possible in order to facilitate detection of angiotropism.

In light of comparable observations of angiotropic tumor spread in cutaneous and conjunctival melanoma, UM may demonstrate similar distinctive micronodules and macronodules developing beyond the primary intraocular melanoma, most often in episcleral tissue sites. Because of the association of the latter nodules with intrascleral angiotropism, we believe that these entities constitute microscopic and macroscopic satellites/in transit metastases. Irrespective of the terms previously employed, these local metastatic foci are almost always predictive of an aggressive and often fatal clinical course $e^{8,17,30-34}$ as in cutaneous and conjunctival melanomas. ${ }^{20,22,23}$

A number of studies have recently shown that molecular studies such as gene expression profiling, loss of chromosome 3, 8q gain, and BAP1 mutation are among the most robust prognostic indicators in UM. ${ }^{3,4,6,7,35}$ However, in our study, angiotropism was also a strong prognostic factor for metastases and death. Given the significance of monosomy 3 and BAP1 mutation in metastases and death ${ }^{3,4,6,7}$ and recent observations that BAP1 mutation may give rise to a poorly differentiated, 'stem-like' phenotype ${ }^{35}$ comparable to what we have observed in angiotropic cutaneous melanoma, ${ }^{12,15}$ the possible relationship between BAP1 mutation and angiotropism in UM merits investigation. However, we found no correlation between angiotropism and BAP1 and monosomy 3 . The absence of such a correlation may potentially be attributed to a number of factors. First of all, there is the matter of missing data, particularly for BAP1 mutation, in a small number of melanomas in our study that may have influenced our results. Another matter is that the detection of angiotropism in UM is subject to the availability of adequate melanoma tissue, ie, enucleation specimens and adequate tissue sampling in tissue specimens. In any case, the relationship between UM angiotropism and various molecular factors such as monosomy 3 and BAP1 mutation requires more detailed study. Provisionally, our results suggest that the angiotropic metastatic pathway may possibly involve other molecular mechanisms. Notably the authors of a recent study describing aggressive skull base metastases from UM discussed the possibility of 'a metastatic pathway independent of BAP1'. ${ }^{36}$

It is of interest to examine angiotropism in relation to other established clinical and histopathological prognostic factors in UM. Although only angiotropism maintained independent predictive value in the multivariate analyses performed for metastases and death, extraocular extension was also predictive of metastasis and tumor diameter and epithelioid cell type predictive of death. In the present chi-square test, angiotropism was associated with both extraocular extension and epithelioid cell phenotype but not tumor diameter. One plausible explanation of this association with epithelioid cell type is that this phenotype correlates with the poorly differentiated stem-cell-like phenotype associated with angiotropic melanoma cell migration. ${ }^{12,15}$ In contrast, while some studies have demonstrated that tumor diameter (TD) in UM is an independent prognostic factor for metastasis and death on multivariate analysis, ${ }^{30,37}$ our results showed that TD remained independent only for death.

The limitations of our study include a relatively small study population of rather advanced enucleation specimens and the fact that angiotropism can only be assessed in enucleations. Increasingly, prognosis in UM may be evaluated by (1) imaging techniques for the measurement of tumor diameter and thickness, and (2) fine-needle biopsy that permits genomic analysis and determination of melanoma cell type, obviating the need for enucleation. It is clear that there are advantages and drawbacks of this relatively noninvasive assessment of prognosis. In any case, the evaluation of angiotropism for prognostic purposes in UM may not be possible in all tumors and cannot be used for guiding the management of primary tumors and the metastatic surveillance in all patients.

Irrespective of the latter drawback, the fundamental value of angiotropism is its importance as a biological phenomenon and marker of progression and metastasis. By angiotropism and EVMM, melanoma cells may spread to nearby or more distant sites. The relationships between cancer and 
Table 7 Summary of current knowledge concerning the molecular basis of tumor cell angiotropism and extravascular migratory metastasis: cancer pathways and potential genes and molecules implicated

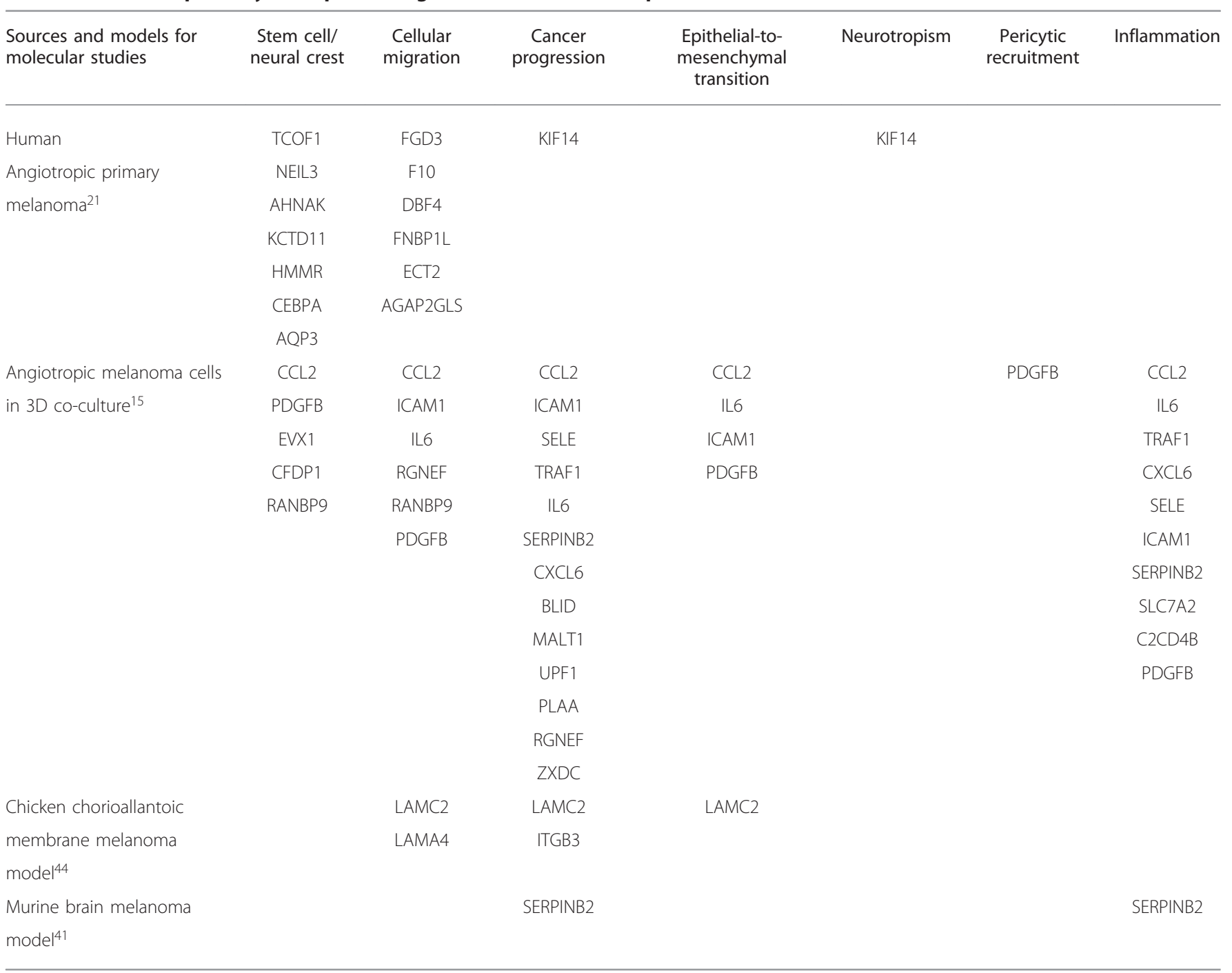

embryogenesis have been recognized for many years. Notably, the embryonic pathways of highly migratory NCC and their regulation during development eventuate in, among other phenotypes, the establishment of melanocytes in the skin and in the uveal tract. ${ }^{38}$ The conspicuous analogies between angiotropism/EVMM and embryonic migration, especially migration of neural crest cells along vessels in the embryo, ${ }^{39,40}$ suggest that angiotropism/EVMM in melanoma may recapitulate stem/embryonic cell migration in order to reach secondary sites. ${ }^{12}$ Interestingly, recent work has demonstrated that vascular co-option, ie, the utilization of preexistent vessels by tumor cells for vital blood supply rather than newly formed vessels via angiogenesis, is closely related if not identical to angiotropism. ${ }^{41}$ Tumor cells implicated in angiotropism and vascular co-option could represent vascular niches comprised of cancer stem cells or cancer cells with stem/embryonic properties ${ }^{41}$ and therefore represent an important therapeutic target in cancer. It has been demonstrated that angiotropic melanoma cells are linked to the endothelium by a modified amorphous basement membrane rich in laminin. ${ }^{12}$ The role of cell-to-cell interactions via cell surface receptors, such as integrins and other laminin-binding proteins, regulate gene expression and significantly influence cell fate. Given the role of laminin in NCC migration, tumor migration, and metastasis, the presence of laminin between melanoma cells and endothelial cells raises the possibility that exposed cryptic promigratory sites on laminin triggers tumor cell motility along the abluminal surface. ${ }^{12}$ Several studies have demonstrated the presence and role of laminins between angiotropic melanoma cells and the endothelium. ${ }^{42}$

During vascular co-option, it has been shown that tumor cells are anchored to the endothelial basement membrane of vascular channels. ${ }^{43}$ Although the molecular mechanisms for these processes remain poorly understood, several studies 
have detected genes/molecules potentially implicated in the migration of angiotropic melanoma cells along vessels. ${ }^{15,21,44}$ Among them, in addition to laminin-related genes, ${ }^{44}$ candidate molecules have been linked to stem cell and neural crest cells, cellular migration, cancer progression, epithelialto-mesenchymal transition, pericytic recruitment, and inflammation ${ }^{15,21}$ (Table 7).

Finally, along with angiotropism, EVMM also connotes migration along other tracks such as nerves (neurotropism) ${ }^{12}$ and a variety of other anatomical structures potentially guiding the invasion of tumor cells for considerable distances. ${ }^{28,45,46}$ Examples include the peritoneum, pleura, and brain ventricles and other structures ensheathed by a basement membrane, such as myofibers and adipocytes. Altogether, these various extravascular routes or EVMM may constitute an alternative mechanism of continuous tumor spread distinct from intravascular dissemination. ${ }^{41}$

The goals of our ongoing investigation at the basic science level are to develop a specific biomarker for angiotropism, to better understand the mechanisms underlying this migratory process, and ultimately to define new targets for limiting tumor growth and metastasis in this commonly fatal disease.

\section{DISCLOSURE/CONFLICT OF INTEREST}

The authors declare no conflict of interest.

1. Correa ZM. Assessing prognosis in uveal melanoma. Cancer Control 2016;23:93-98.

2. Weis E, Salopek TG, McKinnon JG, et al. Management of uveal melanoma: a consensus-based provincial clinical practice guideline. Curr Oncol 2016;23:e57-e64.

3. Cassoux N, Rodrigues MJ, Plancher C, et al. Genome-wide profiling is a clinically relevant and affordable prognostic test in posterior uveal melanoma. Br J Ophthalmol 2014;98:769-774.

4. Worley LA, Onken MD, Person E, et al. Transcriptomic versus chromosomal prognostic markers and clinical outcome in uveal melanoma. Clin Cancer Res 2007;13:1466-1471.

5. Damato B, Eleuteri A, Taktak AF, et al. Estimating prognosis for survival after treatment of choroidal melanoma. Prog Retin Eye Res 2011;30: 285-295.

6. Harbour JW, Onken MD, Roberson ED, et al. Frequent mutation of BAP1 in metastasizing uveal melanomas. Science 2010;330:1410-1413.

7. Decatur $\mathrm{CL}$, Ong E, Garg N, et al. Driver mutations in uveal melanoma: associations with gene expression profile and patient outcomes. JAMA Ophthalmol 2016;134:728-733.

8. Starr HJ, Zimmerman LE. Extrascleral extension and orbital recurrence of malignant melanomas of the choroid and ciliary body. Int Ophtalmol Clin 1962;2:369-384.

9. Shammas HF, Blodi FC. Orbital extension of choroidal and ciliary body melanomas. Arch Ophthalmol 1977;95:2002-2005.

10. Affeldt JC, Minckler DS, Azen SP, et al. Prognosis in uveal melanoma with extrascleral extension. Arch Ophthalmol 1980;98:1975-1979.

11. Coupland SE, Campbell I, Damato B. Routes of extraocular extension of uveal melanoma: risk factors and influence on survival probability. Ophthalmology 2008;115:1778-1785.

12. Lugassy C, Péault B, Wadehra $M$, et al. Could pericytic mimicry represent another type of melanoma cell plasticity with embryonic properties? Pigment Cell Melanoma Res 2013;26:746-754.

13. Bald T, Quast T, Landsberg J, et al. Ultraviolet-radiation-induced inflammation promotes angiotropism and metastasis in melanoma. Nature 2014;507:109-113.

14. Landsberg J, Tüting $\mathrm{T}$, Barnhill $\mathrm{RL}$, et al. The role of neutrophilic inflammation, angiotropism, and pericytic mimicry in melanoma progression and metastasis. J Invest Dermatol 2016;136: 372-377.

15. Lugassy C, Wadehra M, Li X, et al. Pilot study on 'pericytic mimicry' and potential embryonic/stem cell properties of angiotropic melanoma cells interacting with the abluminal vascular surface. Cancer Microenviron 2013;6:19-29.

16. Seddon JM, Albert DM, Lavin PT, et al. A prognostic factor study of disease-free interval and survival following enucleation for uveal melanoma. Arch Ophthalmol 1983;101:1894-1899.

17. Zografos L. Tumeurs Intraoculaires. Masson: Paris, France, 2002; 236-251.

18. Ly LV, Odish OF, Wolff-Rouendaal DD, et al. Intravascular presence of tumor cells as prognostic parameter in uveal melanoma: a 35year survey. Invest Ophthalmol Vis Sci 2010;51:658-665.

19. Barnhill RL, Dy K, Lugassy C. Angiotropism in cutaneous melanoma: a prognostic factor strongly predicting risk for metastasis. J Invest Dermatol 2002;119:705-706.

20. Van Es SL, Colman M, Thompson JF, et al. Angiotropism is an independent predictor of local recurrence and in-transit metastasis in primary cutaneous melanoma. Am J Surg Pathol 2008;32: 1396-1403.

21. Lugassy C, Lazar V, Dessen P, et al. Gene expression profiling of human angiotropic primary melanoma: selection of 15 differentially expressed genes potentially involved in extravascular migratory metastasis. Eur J Cancer 2011;47:1267-1275.

22. Wilmott J, Haydu L, Bagot M, et al. Angiotropism is an independent predictor of microscopic satellites in primary cutaneous melanoma. Histopathology 2012;61:889-898.

23. Barnhill RL, Lemaitre $S$, Lévy-Gabrielle $C$, et al. Satellite in transit metastases in rapidly fatal conjunctival melanoma: implications for angiotropism and extravascular migratory metastasis (description of a murine model for conjunctival melanoma). Pathology 2016;48: 166-176.

24. Histopathologic characteristics of uveal melanomas in eyes enucleated from the Collaborative Ocular Melanoma Study. COMS report no. 6. Am J Ophthalmol 1998;125:745-766.

25. Barnhill RL, Lugassy C. Angiotropic malignant melanoma and extravascular migratory metastasis: description of 36 cases with emphasis on a new mechanism of tumour spread. Pathology 2004;36: 485-490.

26. Barnhill RL, Busam KJ, From L, et al. Inter-observer concordance for the recognition of angiotropism in human melanoma. Pigment Cell Melanoma Res 2011;24:582-583.

27. Kizhatil K, Ryan M, Marchant JK, et al. Schlemm's canal is a unique vessel with a combination of blood vascular and lymphatic phenotypes that forms by a novel developmental process. PLoS Biol 2014;12:e1001912.

28. Friedl P, Alexander S. Cancer invasion and the microenvironment: plasticity and reciprocity. Cell 2011;147:992-1009.

29. Wolter JR. Orbital extension of choroidal melanoma: within a long posterior ciliary nerve. Trans Am Ophthalmol Soc 1983;LXXXI: 48-63.

30. Walter SD, Chao DL, Feuer W, et al. Prognostic implications of tumor diameter in association with gene expression profile for uveal melanoma. JAMA Ophthalmol 2016;134:734-740.

31. Shields CL, Santos MC, Shields JA, et al. Extraocular extension of unrecognized choroidal melanoma simulating a primary optic nerve tumor: report of two cases. Ophthalmology 1999;106: 1349-1352.

32. Sambuelli R, Luna JD, Reviglio VE, et al. Small choroidal melanoma with massive extraocular extension: invasion through posterior scleral emissary channels. Int Ophthalmol 2001;24:213-218.

33. Burnier M, Shields JA. Personal communication. Biannual Meeting of the PanAmerican Society of Ophthalmic Pathology 'Lorenz E. Zimmerman'; 21 July 2001; Sheraton Hotel, Buenos Aires, Argentina.

34. Shields JA, Shields CL. Massive orbital extension of posterior uveal melanoma. J Ophthal Plast Reconstr Surg 1991;7:238-251.

35. Matatall KA, Agapova OA, Onken MD, et al. BAP1 deficiency causes loss of melanocytic cell identity in uveal melanoma. BMC Cancer 2013;13:371.

36. Yonekawa Y, Kim IK, Gragoudas ES, et al. Aggressive skull base metastasis from uveal melanoma: a clinicopathologic study. Eur J Ophthalmol 2014;24:811-813. 
37. Correa ZM, Augsburger JJ. Independent prognostic significance of gene expression profile class and largest basal diameter of posterior uveal melanomas. Am J Ophthalmol 2016;162:20-27.

38. Le Douarin NM, Creuzet $\mathrm{S}$, Couly G, et al. Neural crest cell plasticity and its limits. Development 2004;131:4637-4650.

39. Schwarz $\mathrm{O}$, Maden $\mathrm{CH}$, Vieira JM, et al. Neuropilin 1 signaling guides neural crest cells to coordinate pathway choice with cell specification. PNAS 2009:106:6164-6169.

40. Nagy N, Mwizerwa O, Yaniv K, et al. Endothelial cells promote migration and proliferation of enteric neural crest cells via beta1 integrin signaling. Dev Biol 2009;330:263-272.

41. Bentolila LA, Prakash R, Mihic-Probst D, et al. Imaging of angiotropism/ vascular co-option in a murine model of brain melanoma: implications for melanoma progression along extravascular pathways. Sci Rep 2016;6:23834
42. Lugassy C, Zadran S, Bentolila LA, et al. Angiotropism, pericytic mimicry and extravascular migratory metastasis in melanoma: an alternative to intravascular cancer dissemination. Cancer Microenviron 2014:7:139-152.

43. Szabo V, Bugyik E, Dezso K, et al. Mechanism of tumour vascularization in experimental lung metastases. J Pathol 2015;235: 384-396.

44. Lugassy C, Torres-Muñoz JE, Kleinman HK, et al. Overexpression of malignancy-associated laminins and laminin receptors by angiotropic human melanoma cells in a chick chorioallantoic membrane model. J Cutan Pathol 2009;36:1237-1243.

45. Friedl $\mathrm{P}$, Wolf $\mathrm{K}$. Tumour-cell invasion and migration: diversity and escape mechanisms. Nat Rev Cancer 2003;3:362-374.

46. Clark AG, Vignjevic DM. Modes of cancer cell invasion and the role of the microenvironment. Curr Opin Cell Biol 2015;36:13-22. 